\title{
20001 A Case Report Using Goose Neck Technical Microsnare for Severe Cervical Internal Carotid Artery Occlusion with Dolichoarteriopathy
}

Atsuuji Kuwajima, ${ }^{1}$ Hirotaka Okumura, ${ }^{1}$ Takato Nakajo, ${ }^{1}$ Eisuke Hirose, ${ }^{2}$ Ryo Irie, ${ }^{1}$ Yusuke Kobayashi, ${ }^{1}$ Kazuya Higashizono, ${ }^{3}$ and Tohru Mizutani ${ }^{1}$

Objective: We report the use of a Goose Neck microsnare for cervical internal carotid artery (ICA) occlusion in a patient with dolichoarteriopathy in whom it was difficult to achieve recanalization.

Case Presentation: A 65-year-old woman underwent thrombectomy for a tandem lesion of left M1 occlusion and left cervical ICA occlusion. Recanalization of left M1 occlusion was achieved. For left cervical ICA occlusion, we attempted multiple thrombectomy using an existing device, but a hard clot with mobility was caught due to dolichoarteriopathy, which made thrombectomy difficult. Using a Goose Neck microsnare, we were able to capture the thrombus and achieve recanalization.

Conclusion: Thrombectomy by capturing the thrombus using a Goose Neck microsnare may be useful for capturing hard clots with mobility when it is difficult to achieve recanalization with existing devices.

Keywords > acute ischemic stroke, endovascular treatment, dolichoarteriopathy, Goose Neck microsnare

\section{Introduction}

The usefulness and safety of mechanical thrombectomy for acute ischemic stroke were established by large-scale randomized studies/meta-analyses published after 2015.1) In the American Heart Association/American Stroke Association guidelines, this procedure is recommended as class I. Following recent advances in devices and the development of many combined techniques, a high recanalization rate on

${ }^{1}$ Department of Neurosurgery, Showa University Hospital, Tokyo, Japan

${ }^{2}$ Department of Neurosurgery, AOI Universal Hospital, Kawasaki, Kanagawa, Japan

${ }^{3}$ Department of Neurosurgery, Ebara Hospital, Tokyo Metropolitan Health and Hospitals Corporation, Tokyo, Japan

Received: August 9, 2020; Accepted: December 28, 2020 Corresponding author: Atsuuji Kuwajima. Department of Neurosurgery, Showa University Hospital, 1-5-8 Hatanodai, Shinagawa, Tokyo 142-8666, Japan

Email: atuuji-kuwajima@s5.dion.ne.jp

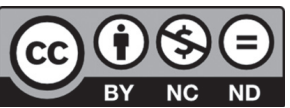

This work is licensed under a Creative Commons Attribution-NonCommercial-

NoDerivatives International License.

(C)2021 The Japanese Society for Neuroendovascular Therapy the first pass was reported. ${ }^{2,3)}$ However, there are patients in whom recanalization is not achieved despite the use of conventional stent retrievers, a direct aspiration first pass technique (ADAPT), or combined techniques due to anatomical vascular anomalies or the characteristics of thrombi.

In this study, we report a patient with cervical internal carotid artery (ICA) occlusion in whom it was difficult to achieve recanalization in the presence of an anomaly of the ICA course, dolichoarteriopathy, and a thrombus was captured using a catheter for removing intravascular foreign bodies, a Goose Neck Microsnare (Medtronic, Minneapolis, MN, USA), leading to recanalization.

\section{Case Presentation}

Patient: A 65-year-old woman.

Complaints: Motor aphasia, and paralysis of the right upper and lower limbs

Medical history: Anxiety neurosis, after surgery for uterine cancer, hypertension, and dyslipidemia

Family history: Not contributory.

Lifestyle: No alcohol consumption. Smoking (5-6 cigarettes/ day for 45 years). 
Kuwajima $A$, et al.

Present illness: Consciousness disorder was noted upon waking and she was transported to a hospital by ambulance at 9:22. Regarding the consciousness level, the Japan Coma Scale (JCS) score was III-100 and the Glasgow Coma Scale (GCS) score was E1V1M5. There was no anisocoria or laterality of the limbs. Head computed tomography (CT) revealed no abnormal findings. She had taken many antipsychotic drugs. By a diagnosis of acute drug addiction, she was admitted to the Department of Internal Medicine. On the same day, paralysis of the right upper and lower limbs developed at 15:00. Head magnetic resonance imaging (MRI)/magnetic resonance angiography (MRA) revealed acute ischemic stroke related to left ICA occlusion and she was referred to our hospital. The final onset-free confirmation time was 19:00 the day before arrival. The interval until arrival to our hospital was 25 hours and 8 minutes.

Physical examination on admission: The height, body weight, blood pressure, pulse, and body temperature were $172 \mathrm{~cm}, 61.4 \mathrm{~kg}, 148 / 90 \mathrm{mmHg}, 71$ times/minute (regular), and $38.5^{\circ} \mathrm{C}$, respectively. Concerning the consciousness level, the JCS score was II-10 and the GCS score was E3V1M6. Paralysis of the right upper and lower limbs was evaluated as $1 / 5$ on the Manual Muscle Test (MMT). The National Institutes of Health Stroke Scale (NIHSS) score was 12 .

Laboratory data on admission: The leukocyte count, hemoglobin $(\mathrm{Hb})$ level, hematocrit $(\mathrm{Ht})$, platelet count, PT-INR, brain natriuretic peptide (BNP) level, and D-dimer level were $7400 / \mu \mathrm{L}, 13.0 \mathrm{~g} / \mathrm{dL}, 36.8 \%, 142000 / \mu \mathrm{L}, 1.05$, $35.7 \mathrm{pg} / \mathrm{mL}$, and $2.19 \mathrm{mg} / \mathrm{dL}$, respectively. Electrocardiography demonstrated sinus rhythm, with a pulse of 73 times.
When reconfirming images from the previous hospital, a hyper-dense middle cerebral artery (MCA) sign at the horizontal segment (M1) of the left MCA was observed on head CT. Both diffusion-weighted images (DWIs) and fluid-attenuated inversion recovery (FLAIR) images on head MRI confirmed high signal intensity involving the left temporal gyrus to frontal/temporal lobes. The DWI-Alberta Stroke Program Early Computed Tomography Score (DWI-ASPECTS) was 6/10 (Fig. 1A). On head MRA and three-dimensional (3D) CT angiography (CTA), which were performed at our hospital, the left ICA origin was occluded. The ICA distal to the posterior communicating artery was visualized and a thrombus-related shadow defect was observed at the left M1 segment (Fig. 1B). Torsion of the contralateral ICA was marked, suggesting marked torsion of the affected-side ICA (Fig. 1C). Although the interval from the final onset-free confirmation time was $\geq 24$ hours, endovascular treatment was performed for the following reasons: the left M1 segment was partially occluded and cross-flow from the anterior communicating artery was expected; there was a dissociation between the clinical symptoms and imaging findings, and a clinical-DWI mismatch.

Endovascular treatment: Under local anesthesia, a 9-Fr sheath introducer was inserted into the right femoral artery and heparin at 5000 units was administered for systemic heparinization. A 9-Fr Optimo (Tokai Medical Products, Aichi, Japan) was inserted into the left common carotid artery (CCA). Angiography demonstrated occlusion on the peripheral side of the cervical ICA. There was no stenotic or dissecting lesion at the origin of the left ICA (Fig. 2A). Furthermore, the intracranial left

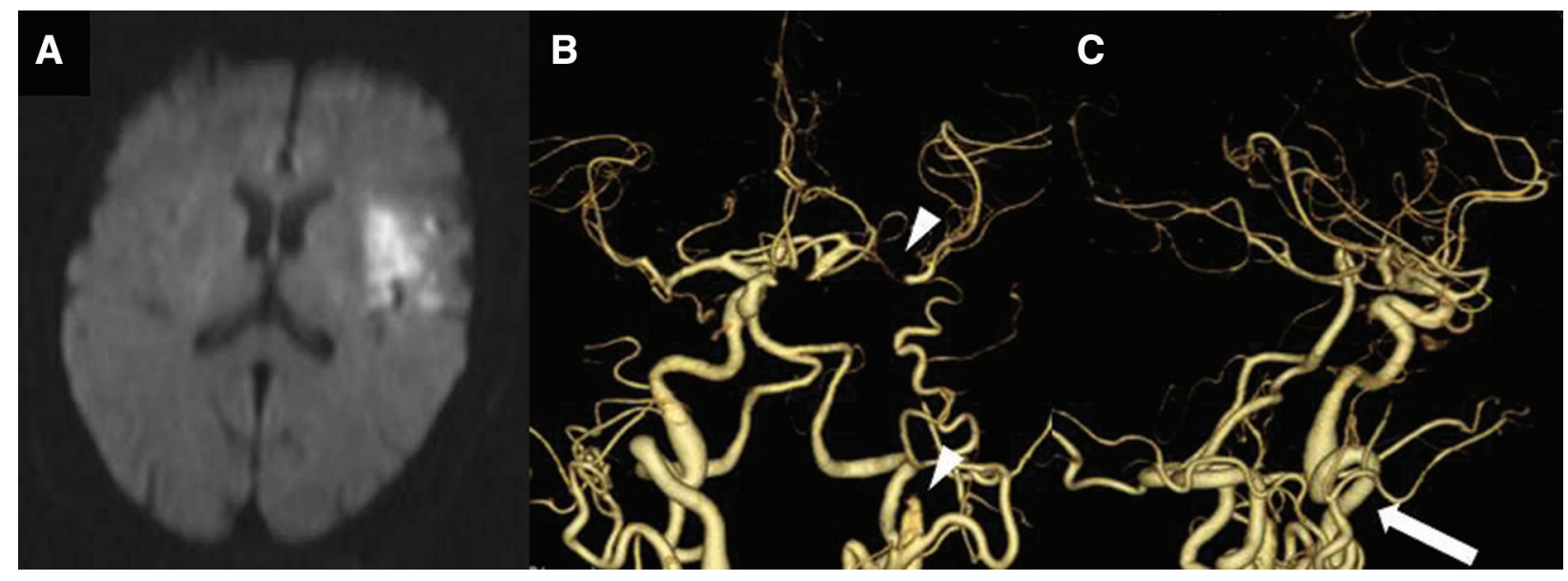

Fig. 1 (A) Ischemic lesions were visualized as high-intense lesions in the left MCA-perfused territory (insular cortex and frontal lobe and temporal lobe) on diffusion-weighted imaging. (B) The left MCA and
ICA was occluded on 3D-CTA (arrowheads). (C)The right cervical ICA had coiling dolichoarteriopathy with a circular structure on 3D-CTA (arrow). MCA: middle carotid artery; ICA: internal carotid artery 


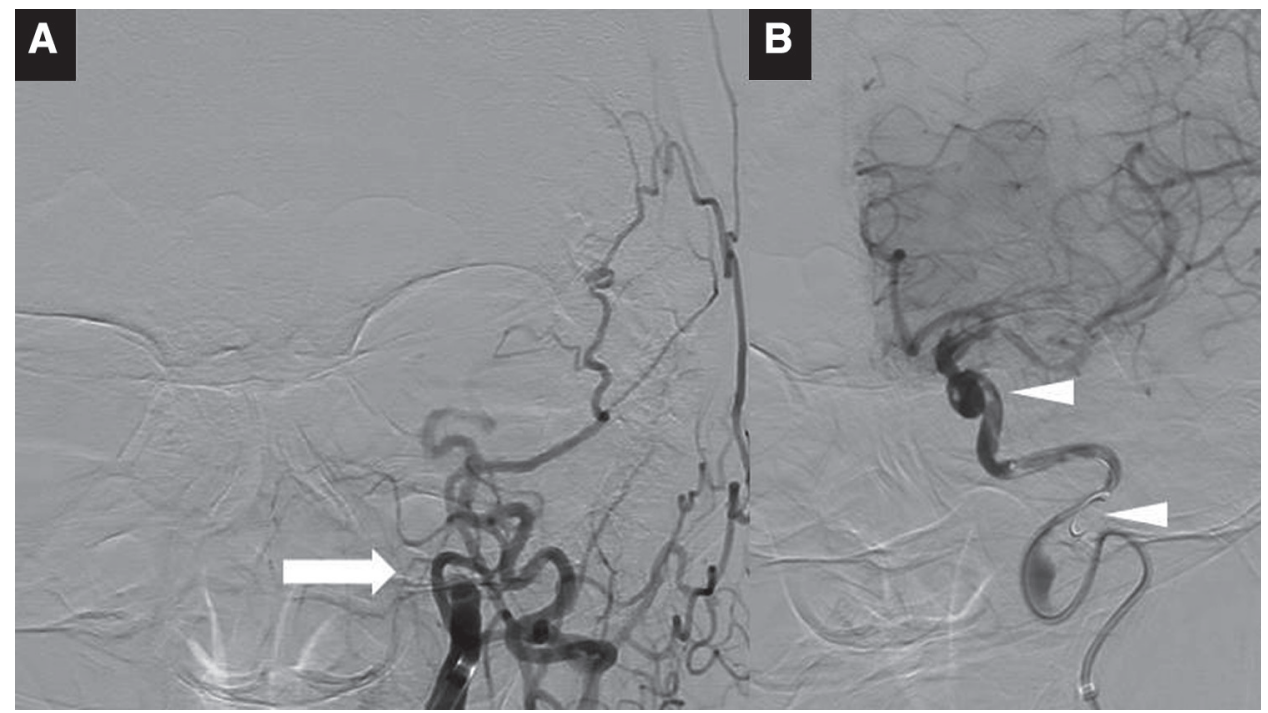

Fig. 2 Antero-posterior view. (A) The left cervical ICA was occluded on angiography (arrow). (B) Recanalization of the left MCA was achieved by SR. Thrombi were found in the left ICA (C4-5 segment and petrous portion and cervical portion) on angiography (arrowheads). ICA: internal carotid artery; MCA: middle carotid artery; SR: stent retriever

ICA was contrast-enhanced through a collateral pathway via the left ophthalmic artery from the left external carotid artery (ECA). A tandem lesion consisting of thrombi involving the left cervical ICA to $\mathrm{C} 3$ segment of the intracranial ICA and at the left M1 segment was detected. Initially, a retrograde approach for thrombectomy through the intracranial left M1 segment was selected.

The 9-Fr Optimo was guided to the left ICA origin and manual aspiration was performed. However, no thrombus was able to be aspirated. Using a Penumbra ACE60 (Penumbra, Inc., Alameda, CA, USA) (ACE60) as an intermediate catheter, a Marksman (eV3; Covidien, Irvine, CA, USA) and Traxecess 14 with a J-shaped tip (Terumo, Tokyo, Japan) were slowly guided, considering tortuous blood vessels. Lesion crossing at the thrombus site of the left M1 segment was conducted and the ACE60 was elevated to the IC top. A Solitaire Platinum $6 \times 40 \mathrm{~mm}$ (eV3; Covidien) was deployed in the M2 to M1 segments, and the second pass led to intracranial recanalization (24 minutes after puncture) (Fig. 2B). Subsequently, thrombectomy for thrombi of the C4-5 segment of the left ICA and petrous part to the cervical ICA was performed. To remove thrombi of the C4-5 segment of the left ICA, the ACE60 was elevated to the petrous part and a Solitaire Platinum $6 \times 40 \mathrm{~mm}$ was deployed in the $\mathrm{C} 3$ to $\mathrm{C} 5$ segments of the left ICA. The second pass led to recanalization. Dolichoarteriopathy of the cervical ICA with a round structure was present, and a mobile, hard, large thrombus involving this structure was detected. The site of dolichoarteriopathy of the petrous part to the cervical ICA was divided into three segments: Segment A, flexed area of the petrous part to cervical ICA; Segment B, flexed area of the cervical ICA (upward); and Segment $C$, flexed area of the cervical ICA (downward) (Fig. 3A-3D). To remove the thrombus in Segment A, a Solitaire Platinum $6 \times 40 \mathrm{~mm}$ was deployed (1 pass) and a combined technique with the Solitaire Platinum $6 \times 40 \mathrm{~mm}$ and ACE60 was performed (1 pass). However, the thrombus was unable to be retrieved. ADAPT with the ACE60 (2 passes) facilitated transfer of the thrombus to Segment C, but capturing was affected at the site of flexion, making retrieval impossible. In addition, the Solitaire Platinum $6 \times 40 \mathrm{~mm}$ was deployed (4 passes), but the thrombus migrated between Segments B and C, finally reaching Segment C. However, it was unable to be retrieved.

To obtain a strong thrombus-capturing force, a Goose Neck Microsnare $7 \mathrm{~mm}$ was deployed with an attached catheter from the distal side of the Segment $C$ thrombus by removing the ACE60 (Fig. 4A), and the thrombus was retrieved by covering and capturing it (Fig. 4B and 4C). On the first pass, a portion of the thrombus was retrieved (Fig. 4D). On the third pass, the giant, hard thrombus was firmly captured and retrieved into a 9-Fr Optimo. An approximately $10-\mathrm{mm}$ thrombotic mass was extirpated from the 9-Fr Optimo. Recanalization was achieved and the procedure was completed with a Thrombolysis in Cerebral Infarction (TICI) grade of $2 \mathrm{~b}$ (2 hours and 9 minutes after puncture) (Fig. 5A and 5B). 


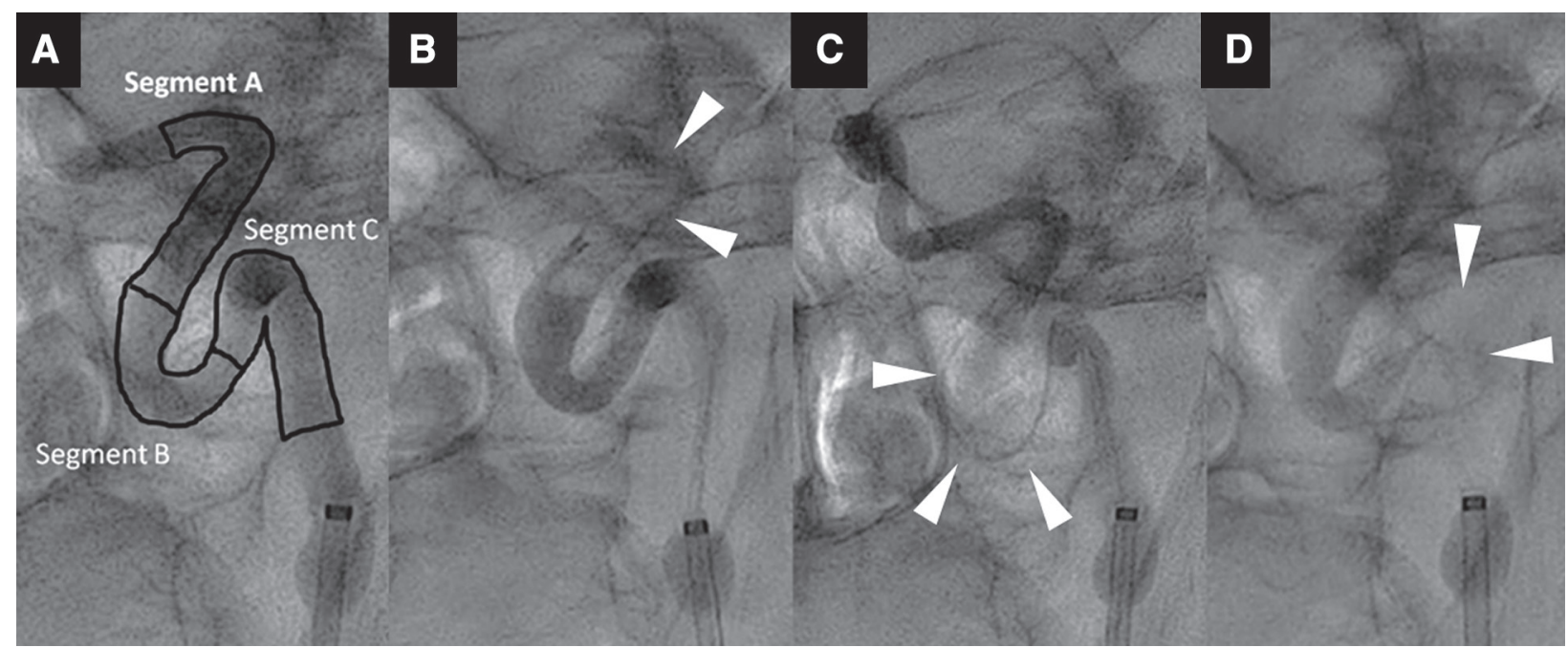

Fig. 3 Antero-posterior view. (A) The lesion from the petrous ICA to dolichoarteriopathy of the cervical ICA was divided into three parts (A-D). (B) Segment A: A thrombus was found at the flexion from the petrous ICA to the cervical ICA (arrowheads). The large thrombus was aspirated with ACE60. (C) Segment B: A thrombus was found at the flexion of the cervical ICA (upward) (arrowheads). A J-shaped Traxecess14 passed through a large thrombus. (D) Segment C: A thrombus was found at the flexion of the cervical ICA (downward) (arrowheads). ICA: internal carotid artery

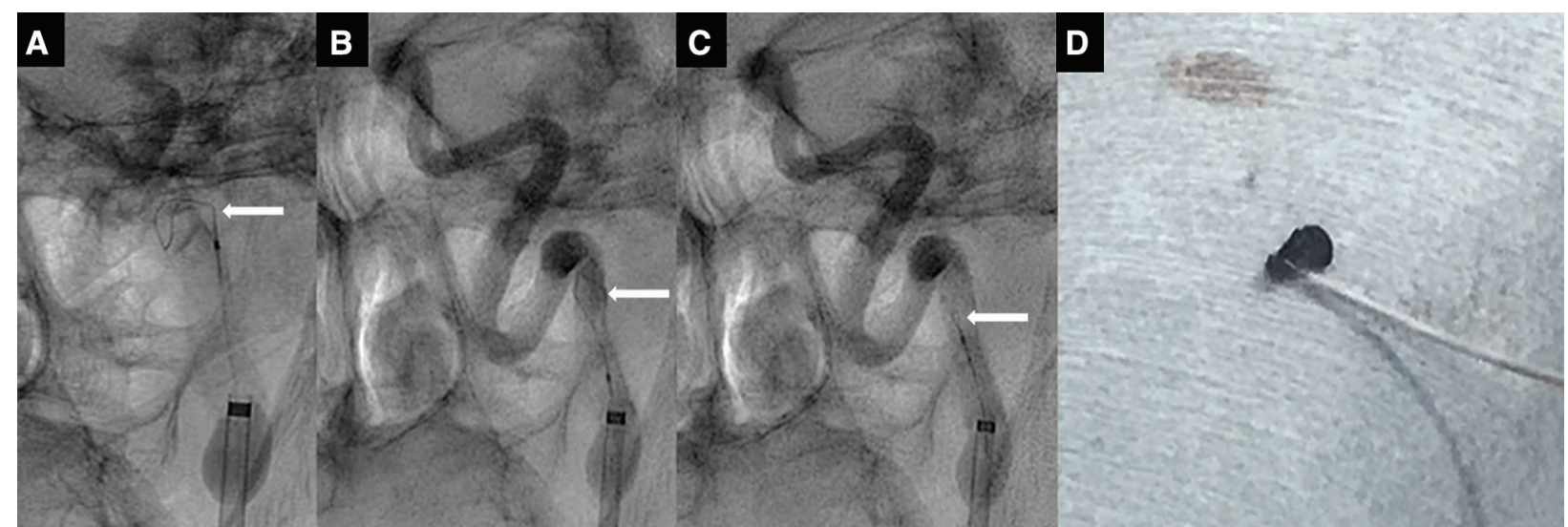

Fig. 4 (A) A Goose Neck microsnare was deployed distal to the thrombus (arrow). (B) The Goose Neck microsnare was placed on the thrombus (arrow). (C) The Goose Neck microsnare captured the

Pathological findings: The extirpated thrombus was mixed containing erythrocytes primarily consisting of fibrin components. Neutrophils and inflammatory cells, such as nuclear destruction, were observed. There was no atheroma or tumor component.

Postoperative course: Postoperative head MRI revealed no increase in the extent of the fresh infarcted focus. Head MRA confirmed recanalization. To investigate the source of embolism, detailed systemic examination was conducted, but there was no significant finding. By a diagnosis of embolic stroke of undetermined source (ESUS), the administration of aspirin at $100 \mathrm{mg} /$ day was started and a Reveal LINQ was inserted. Motor aphasia and paralysis of the right upper and lower limbs gradually reduced from 4 thrombus (arrow). (D) The Goose Neck microsnare captured part of the large thrombus.

days after surgery. The modified Rankin Scale (mRS) score 19 days after surgery was 2 . Concerning the consciousness level, the GCS score was E4V5M6, and paralysis of the right upper and lower limbs was evaluated as MMT 5-/5. The NIHSS score was 4 . The patient was referred to a rehabilitation hospital. After 8 months, no delayed morphological abnormality at the site of dolichoarteriopathy was observed on 3D-CTA.

\section{Discussion}

A high recanalization rate after mechanical thrombectomy for acute ischemic stroke has been achieved with recent advances in devices, salvage therapy, and the development 


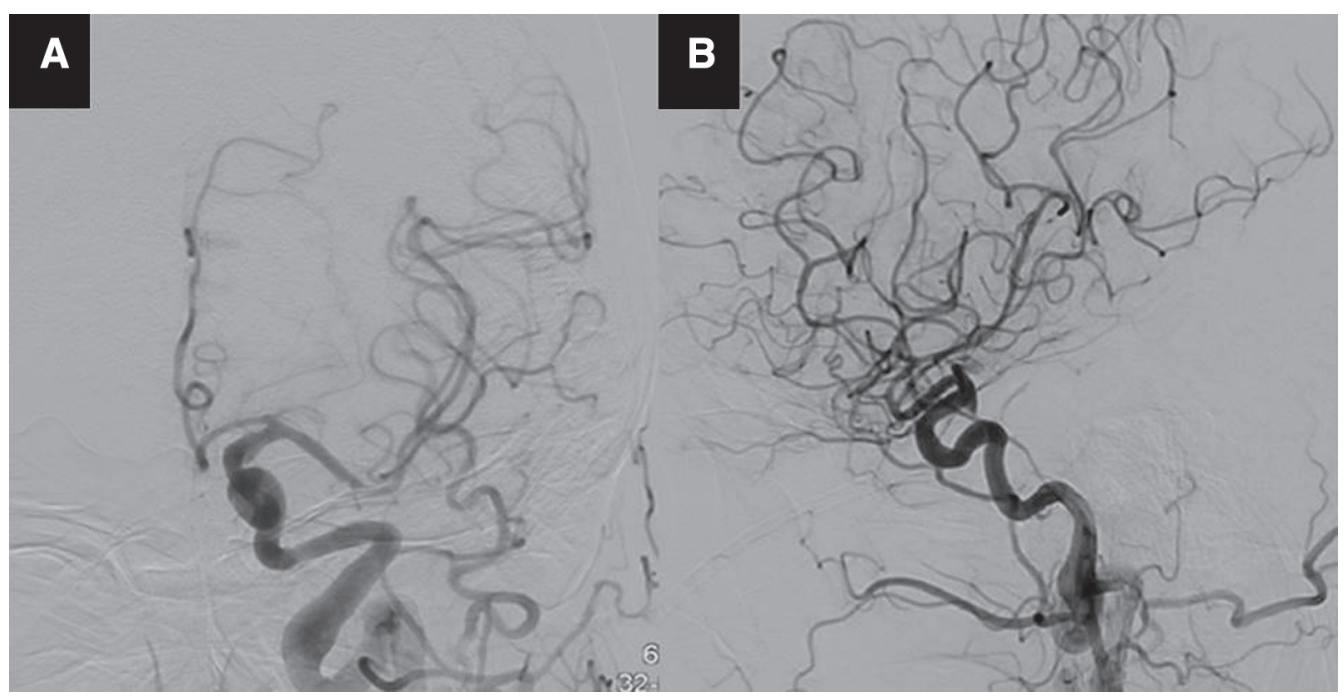

Fig. 5 (A)(B) Recanalization was achieved, classified as $\mathrm{TICI} 2 \mathrm{~B}$ on the final angiography. TICl: Thrombolysis in Cerebral Infarction

of many combined techniques. However, in clinical practice, there are patients in whom it is difficult to achieve recanalization. As factors for poor recanalization, anatomical vascular anomalies (bovine type, aortic arch type II/III, and ICA dolichoarteriopathy), sites of occlusion (cervical ICA, intracranial ICA other than T occlusion, and M2), marked stenosis of the cervical ICA, large-volume thrombi, tandem lesions, hard thrombi, non-embolic features, rare stroke type, overtime medical service, female sex, a history of hypertension, and treatment for many hours have been reported. ${ }^{4-7)}$

In the present case, the second pass led to recanalization at the site of M1 occlusion, but a hard, giant thrombus involved the area of dolichoarteriopathy with a round structure at the site of cervical ICA occlusion, making thrombectomy difficult. At the origin of the ICA, there was no arteriosclerotic or dissecting lesion, but dolichoarteriopathy of the ICA and the hard thrombus affected recanalization.

Vascular course abnormalities related to abnormal ICA development are termed dolichoarteriopathies of the internal carotid artery (DICAs). Metz and Weibel et al. classified DICAs into three types (tortuous, coiling, and kink). ${ }^{8-10)}$ Coiling-type DICAs include C-shaped, S-shaped, U-shaped, and double circular structures, in addition to a round structure, ${ }^{8-10)}$ as demonstrated in the present case. Unilateral or bilateral DICAs are observed in approximately $10 \%-40 \%$ of patients. In particular, the incidence of coiling-type DICAs is the lowest (approximately $3 \%-7 \%)^{7,11,12)}$ Regarding the development of cerebral infarction related to DICAs, a kink-type DICA patient with
ICA stenosis was presented. ${ }^{13)}$ Snelling et al. ${ }^{7)}$ found that vascular torsion related to DICAs was associated with prolongation of the interval until recanalization in the acute phase and a poor prognosis.

The thrombus as an etiological factor in the present case was mobile, hard, and large. Pathologically, a fibrindominant thrombus was suggested. Inflammatory cells with neutrophils were detected. Fibrin-dominant thrombi are hard, with a high coefficient of friction, ${ }^{14)}$ and the recanalization rate is lower than that in patients with red blood cell (RBC)-dominant thrombi. ${ }^{15}$ )

For mechanical thrombectomy, a Solitaire has been used as a first-choice device at our hospital. However, in the present case, it was possible to capture and transfer the hard thrombus, but it was unable to be captured at the site of DICAs; retrieval was not possible. In the package inserts, it is recommended that a Solitaire Platinum $6 \times 40 \mathrm{~mm}$ be indicated for a vascular diameter of $3.0-5.5 \mathrm{~mm}$. In the present case, the extracranial ICA diameter was $6.2-6.8 \mathrm{~mm}$; sufficient adhesion to the vascular wall may not have been achieved. Concerning the hard-thrombus-retrieving capacity, Machi et al. investigated 10 types of stent retrievers using an in vitro vascular model, and reported that white thrombi measuring $\geq 6 \mathrm{~mm}$ were unable to be retrieved because sufficient stent deployment was impossible, allowing a stent to pass through the side of the thrombus. ${ }^{16)}$ Furthermore, Kaneko et al. conducted an in vitro study and found that the segment of a stent retriever was squashed upon stretching it in a markedly flexed blood vessel, making it impossible to capture a thrombus and reducing the recanalization rate, 
Kuwajima $A$, et al.

suggesting that the use of a stent retriever is inappropriate. They reported that the segments of an Embotrap (Cerenovus, Galway, Ireland) and Versi Retriever (NeuroVasc Technologies, Laguna Hills, CA, USA), which have a segmented design, remained even in a markedly flexed blood vessel, improving the recanalization rate. ${ }^{17)}$

In the present case, ADAPT was also performed to treat embolism of the cervical ICA, but the thrombus captured with an ACE60 came off at the site of DICA, making retrieval impossible. Mascitelli et al. compared patients in whom ADAPT was successful with those in whom it was unsuccessful, and reported that factors for poor recanalization after ADAPT included a larger number of flexed blood vessels, larger vascular diameter, vascular diameter greater than that of a catheter, and a low clot burden score as an index for evaluating thrombus-occluded blood vessels. ${ }^{18)}$ In the present case, an ACE60 was selected to eliminate ledge effects, emphasizing its guiding property in flexed blood vessels. Considering the inner diameter of a catheter at its tip alone, that of an ACE60 is $1.8 \mathrm{~mm}$, and that of a Penumbra ACE68 (Penumbra Inc.) (ACE68) is $2.03 \mathrm{~mm}$. A previous study demonstrated that the aspiration force of the latter increased by $25 \% .{ }^{19)}$ Switching to a larger-diameter catheter, such as an ACE68, may also have been a treatment option.

Goose Neck microsnares are used to retrieve and remove intravascular foreign bodies such as mis-inserted embolic substances, damaged catheter/guidewire pieces, pacing leads that fell off, inferior vena cava filters, and metallic stents. However, their use for mechanical thrombectomy was also reported before the introduction of a MERCI retriever (Concentric Medical, Mountain View, CA, USA). Rentzos et al. presented the results of mechanical thrombectomy in 156 patients in whom Goose Neck microsnares were selected as a first-choice device. According to their report, modified TICI $2 \mathrm{~b}$ or higher recanalization was achieved in $74 \%$ of the patients, and the mRS score was 0-2 in 42\%; a favorable outcome was achieved. There was no snare-associated complication. ${ }^{20)}$

The size of Goose Neck microsnares is 2, 4, and $7 \mathrm{~mm}$. In the present case, the ICA diameter was $6.2-6.8 \mathrm{~mm}$, and a slightly larger size, $7 \mathrm{~mm}$, was selected. An attached 0.021 -inch microcatheter was used. For the use of this microsnare, a loop was deployed at an area distal to the thrombus and slowly pulled such that the thrombus was guided into the snare loop. Subsequently, the above microcatheter was guided to capture the snare. In procedures with a snare, it is difficult to accurately evaluate and grasp thrombi. In the present case, all thrombi were grasped on the third pass. Unless a thrombus is hard to some degree, it may be difficult to grasp. Thrombus rupture related to excessive traction by a snare may cause embolization of new territory (ENT); caution is needed.

In the future, issues, such as anatomical vascular anomalies and thrombus properties, may be overcome by the introduction of new devices, improving the recanalization rate. In some patients with mobile, hard thrombi in whom recanalization was not achieved using conventional devices, mechanical thrombectomy with a Goose Neck microsnare may be effective.

\section{Conclusion}

The use of a Goose Neck microsnare for cervical ICA occlusion with DICAs led to recanalization. Mechanical thrombectomy with a Goose Neck microsnare for thrombus grasping may be effective in patients with mobile, hard thrombi in whom it is difficult to achieve recanalization using conventional devices.

\section{Disclosure Statement}

Hirotaka Okumura received rewards, such as lecture fees, from Medtronic Japan Co., Ltd. The other authors declare no conflicts of interest.

\section{References}

1) Goyal M, Menon BK, van Zwam WH, et al: Endovascular thrombectomy after large-vessel ischaemic stroke: a meta-analysis of individual patient data from five randomised trials. Lancet 2016; 387: 1723-1731.

2) Brehm A, Maus V, Tsogkas I, et al: Stent-retriever assisted vacuum-locked extraction (SAVE) versus a direct aspiration first pass technique (ADAPT) for acute stroke: data from the real-world. BMC Neurol 2019; 19: 65.

3) Goto $\mathrm{S}$, Ohshima $\mathrm{T}$, Ishikawa $\mathrm{K}$, et al: A stent-retrieving into an aspiration catheter with proximal balloon (ASAP) technique: a technique of mechanical thrombectomy. World Neurosurg 2018; 109: e468-e475.

4) Kim BM: Causes and solutions of endovascular treatment failure. J Stroke 2017; 19: 131-142.

5) Goda T, Oyama N, Kitano T, et al: Factors associated with unsuccessful recanalization in mechanical thrombectomy for acute ischemic stroke. Cerebrovasc Dis Extra 2019; 9: 107-113. 
6) Flottmann F, Broocks G, Faizy TD, et al: Factors associated with failure of reperfusion in endovascular therapy for acute ischemic stroke: a multicenter analysis. Clin Neuroradiol 2020 Feb 17. doi: 10.1007/s00062-020-00880-8. [Epub ahead of print]

7) Snelling BM, Sur S, Shah SS, et al: Unfavorable vascular anatomy is associated with increased revascularization time and worse outcome in anterior circulation thrombectomy. World Neurosurg 2018; 120: e976-e983.

8) Weibel J, Fields WS: Tortuosity, coiling, and kinking of the internal carotid artery. II. Relationship of morphological variation to cerebrovascular insufficiency. Neurology 1965; 15: 462-468.

9) Weibel J, Fields WS: Tortuosity, coiling, and kinking of the internal carotid artery. I. Etiology and radiographic anatomy. Neurology 1965; 15: 7-18.

10) Metz H, Murray-Leslie RM, Bannister RG, et al: Kinking of the internal carotid artery. Lancet 1961; 1: 424-426.

11) Schenk $P$, Temmel A, Trattnig $S$, et al: Current aspects in diagnosis and therapy of carotid artery kinking. HNO 1996; 44: 178-185. (in German)

12) Benson JC, Brinjikji W, Messina SA, et al: Cervical internal carotid artery tortuosity: A morphologic analysis of patients with acute ischemic stroke. Interv Neuroradiol 2019: 26: 216-221.

13) Wang LJ, Wang DM, Liu JC, et al: Endovascular management of symptomatic carotid stenosis combined with kinking. Zhonghua Wai Ke Za Zhi 2011; 49: 105-108. (in Chinese)
14) Gunning GM, McArdle K, Mirza M, et al: Clot friction variation with fibrin content; implications for resistance to thrombectomy. J Neurointerv Surg 2018; 10: 34-38.

15) Brinjikji W, Duffy S, Burrows A, et al: Correlation of imaging and histopathology of thrombi in acute ischemic stroke with etiology and outcome: a systematic review. J Neurointerv Surg 2017; 9: 529-534.

16) Machi P, Jourdan F, Ambard D, et al: Experimental evaluation of stent retrievers' mechanical properties and effectiveness. J Neurointerv Surg 2017; 9: 257-263.

17) Kaneko N, Komuro Y, Yokota H, et al: Stent retrievers with segmented design improve the efficacy of thrombectomy in tortuous vessels. J Neurointerv Surg 2019; 11: 119-122.

18) Mascitelli JR, Kellner CP, Oravec CS, et al: Factors associated with successful revascularization using the aspiration component of ADAPT in the treatment of acute ischemic stroke. J Neurointerv Surg 2017; 9: 636-640.

19) Delgado Almandoz JE, Kayan $Y$, Wallace AN, et al: Larger ACE 68 aspiration catheter increases first-pass efficacy of ADAPT technique. J Neurointerv Surg 2019; 11: 141-146.

20) Rentzos A, Lundqvist C, Karlsson JE, et al: Mechanical embolectomy for acute ischemic stroke in the anterior cerebral circulation: the Gothenburg experience during 20002011. AJNR Am J Neuroradiol 2014; 35: 1936-1941. 